\title{
Papel do Vocabulário para as Habilidades de Compreensão Leitora
}

\author{
Silvia Brilhante Guimarães' \\ Renata Mousinho ${ }^{2}$ \\ ${ }^{1}$ Universidade Salgado de Oliveira, Niterói, RJ \\ ${ }^{2}$ Universidade Federal do Rio de Janeiro, Rio de Janeiro, RJ
}

\begin{abstract}
Resumo
O presente estudo explorou a relação da medida de vocabulário da Escala WISC-III com as habilidades de precisão de leitura de palavras e de compreensão de texto, com 174 crianças, matriculadas no $3^{\circ}$ e $4^{\circ}$ anos do ensino fundamental. Identificou-se evidência de predição do vocabulário na compreensão de texto, mesmo depois do controle da precisão de leitura de palavras, da consciência fonológica e morfológica, e da memória de curto prazo. O mesmo não foi encontrado para a leitura de palavras. Os resultados indicam que o vocabulário medido pelo teste do WISC-III se relaciona com as habilidades de leitura, mas tem papel preditivo apenas na compreensão de texto. Esses achados são discutidos à luz da teoria do Modelo Simples de Leitura e da Hipótese Qualidade Lexical.

Palavras-chave: semântica, leitura de palavras, compreensão de texto
\end{abstract}

\section{Role of Vocabulary for Reading Comprehension Skills}

\begin{abstract}
The present study explored the relationship of the WISC-III Vocabulary measure with the skills of word reading and reading comprehension in 174 children enrolled in the 3rd and 4th grades of Elementary School. Evidence of prediction of vocabulary in reading comprehension was identified, even after controlling for word reading, phonological awareness, short-term memory, and morphological awareness. The same was not found for the skill of word reading. The results indicate that the WISC-III vocabulary relates to reading skills, but has a predictive role only in text comprehension. These findings are discussed in light of the Simple Reading Model theory and the Lexical Quality Hypothesis.
\end{abstract}

Keywords: Semantics; reading of words; reading comprehension

Papel del Vocabulario para las Habilidades de Comprensión Lectora

\begin{abstract}
Resumen
En el presente estudio fue analizada la relación de la medida de vocabulario de la Escala WISC-III con las habilidades de comprensión de lectura y de texto, participando 174 niños, matriculados en $3^{\circ}$ y $4^{\circ}$ año de Enseñanza Primaria. Se encontraron evidencias de predicción de vocabulario en la comprensión de texto, inclusive después de controlar otras variables como precisión lectora de palabras, conciencia fonológica, memoria de corto plazo y conciencia morfológica. La misma evidencia no fue encontrada en la lectura de palabras. Los resultados indican que el vocabulario medido por el WISC-III se relaciona con las habilidades de lectura, pero tiene un papel predictivo sólo en la comprensión de texto. Estos hallazgos son discutidos a la luz de la teoría del Modelo de Lectura Simple y la Hipótesis Calidad Léxica.

Palabras clave: semántica; lectura de palabras; comprensión de texto
\end{abstract}

A ideia de que o conhecimento dos significados das palavras faz parte do processo da leitura não é nova. Estudos internacionais, há muito tempo, mostram que o vocabulário está fortemente relacionado à leitura (Anderson \& Freebody, 1981; Beck, Perfetti, \& McKeown, 1982; Stanovich, Cunningham, \& Feeman, 1984). No entanto, a maior parte das pesquisas nacionais centrou-se no papel vital que as habilidades fonológicas desempenham no desenvolvimento da leitura, tendo havido poucos estudos verificando os aspectos semânticos, como o vocabulário (Maluf, Zanella, \& Pagnez, 2006; Santos, Melo, \& Roazzi, 2016). Neste estudo, considera-se o papel potencial do vocabulário para o desenvolvimento da leitura. Especificamente, busca-se investigar como essa habilidade verbal, medida pelo subteste de vocabulário da Escala Wechsler Intelligence Scales for Children- III (WISC-III), relaciona-se com as medidas de leitura: precisão de leitura de palavras e compreensão textual via teste CLOZE.

O vocabulário é definido como o conhecimento dos significados das palavras pela via da linguagem oral ou escrita, de maneira expressiva (produzindo palavras) e receptiva (compreendendo palavras). Os pesquisadores sugerem que o vocabulário está representado em diferentes dimensões na memória do leitor, isto é, na amplitude e profundidade (Oakhill, Cain, \& Eldro, 2015; Ouellette, 2006). A amplitude do vocabulário diz respeito ao tamanho do léxico mental do leitor, ou seja, 
ao número de palavras que têm algum nível de significado para o indivíduo (Anderson \& Freebody, 1981). A profundidade do vocabulário refere-se à riqueza de conhecimento que o indivíduo possui sobre palavras conhecidas, ou seja, a extensão da representação semântica (Beck et al., 1982).

O subteste de vocabulário da Escala WISC-III pode ser considerado uma medida de vocabulário que reflete um conhecimento mais profundo dos significados das palavras, ou seja, o quão bem a criança conhece uma determinada palavra. A avaliação desse teste requer da criança o conhecimento que já possui sobre os significados das palavras, conceituando-a oralmente de forma lógica (Wechsler, 2002). Essa medida é importante para a leitura, pois envolve conceituação de palavras, memória semântica (memória de longo prazo) e organização da linguagem.

A relação do vocabulário com a leitura parece lógica. Um dos motivos é que quanto mais se lê, mais palavras são aprendidas a partir do contexto, refinando-se, por conseguinte, o vocabulário em amplitude (quantidade) e profundidade. A criança desenvolve um conhecimento complexo e rico dos significados das palavras em diferentes contextos (Oakhill et al., 2015; Stanovich et al., 1984). O contrário também acontece, quanto maior o nível de vocabulário, mais há possibilidades de compreensão da leitura. Há reciprocidade no desenvolvimento do vocabulário e da compreensão de leitura, tendo cada um efeito benéfico sobre o outro (Verhoeven, van Leeuwe, \& Vermeer, 2011).

Os estudos científicos com diferentes delineamentos apontam a conexão do vocabulário com leitura (Beck et al., 1982; Holahan et al., 2016; Ouellette, 2006; Ouellette \& Beers, 2010; Perfetti \& Stafura, 2014; Verhoeven \& van Leeuwe, 2008; Verhoeven et al., 2011) e em leitores com dificuldade (Nation \& Snowling, 1998; Ricketts, Nation, \& Bishop, 2007). O estudo de metanálise de Elleman, Lindo, Morphy e Compton (2009) mostra que a intervenção do vocabulário têm mostrado efeitos positivos na compreensão de texto, principalmente em compreendedores menos habilidosos.

O papel potencial do vocabulário tem sido investigado dentro de dois modelos teóricos de compreensão de leitura, o Modelo Simples da Leitura (SL) e a Hipótese de Qualidade Lexical (HQL). A teoria simples de leitura de Gough e Tunmer (1986) define a compreensão de leitura como produto de dois componentes: a compreensão linguística e a capacidade de reconhecer ou decodificar palavras. A compreensão linguística envolve todas aquelas habilidades necessárias para compreender um texto falado. Elas vão, desde as habilidades de compreensão e raciocínio da linguagem, até a habilidade verbal ou cognitiva. O reconhecimento ou a decodificação de palavras representam a capacidade de traduzir a palavra escrita em sons, isto é, em fonemas ou sílabas (Hoover \& Gough, 1990).

Ambas habilidades, o reconhecimento de palavras e a compreensão linguística, explicam a maioria da variância da compreensão de texto dos leitores dos ensinos fundamental e médio (Kim \& Wagner, 2015; Foorman et al., 2015). Todavia, elas estão sujeitas às mudanças ao longo do tempo (idade e nível de escolarização) da criança. À medida que as crianças aprendem a ler palavras, o fator limitante na compreensão da leitura muda; do reconhecimento de palavras para a compreensão da linguagem falada (Hoover \& Gough, 1990).

Nos anos iniciais de escolarização, a compreensão de texto é explicada, em grande parte, pela decodificação, porque as crianças precisam entender o princípio alfabético e, com isso, construir rotas de leitura de palavras (Verhoeven \& van Leeuwe, 2008). Com a automatização da decodificação, as crianças reconhecem e pronunciam os significados das palavras imediatamente sem gastar qualquer atenção ou esforço. Isso faz com que, ao longo da escolarização, a compreensão linguística seja um crescente diferenciador entre bons e pobres compreendedores (Hoover \& Gough, 1990).

Dentro da teoria do modelo teórico Simples de Leitura, estudos vêm tentando compreender o papel do vocabulário na contribuição da decodificação ou compreensão linguística (Braze, Tabor, Shankweiler, \& Mencl, 2007; Braze et al., 2016; Tunmer \& Chapman, 2012). Braze et al. (2007) apresentaram evidências de que o vocabulário do leitor adulto (16 a 24 anos) teria um caminho único dentro desse modelo. $\mathrm{O}$ vocabulário afetaria a compreensão textual, independentemente da compreensão linguística e do reconhecimento de palavras. Os autores propuseram uma visão estendida do modelo simples de leitura, no qual incluíram o efeito direto e separado do vocabulário na compreensão de texto, além dos efeitos diretos suportados da decodificação e da compreensão linguística. Todavia, estudos subsequentes (Braze et al., 2016; Tunmer \& Chapman, 2012) não apoiaram a versão de Braze, Tabor, Shankweiler e Mencl, (2007) e mostram não haver necessidade de colocar um efeito direto e separado do vocabulário no modelo Simples de Leitura.

No estudo de Tunmer e Chapman (2012), os pesquisadores exploraram essa questão em crianças do $3^{\circ}$ ano escolar da Nova Zelândia. A medida de vocabulário 
foi um teste de amplitude (PPVT- Peabody Picture Vocabulary Test). Primeiramente, os pesquisadores realizaram uma análise de regressão hierárquica, como em Braze et al. (2007), e encontraram uma contribuição independente do vocabulário na compreensão de texto. $\mathrm{O}$ vocabulário explicou $2,2 \%$ da compreensão de texto depois do controle da idade, da compreensão linguística, do reconhecimento de palavras. No entanto, tal ideia se mostrou fraca, na medida em que os pesquisadores realizaram outra análise estatística fatorial e de modelação estrutural. $\mathrm{Na}$ análise fatorial, o vocabulário se agrupou com a compreensão linguística, em vez de formar seu próprio fator independente, como sugeriram Braze et al. (2007).

Braze et al. (2016) encontraram evidências similares às de Tunmer e Chapman (2012), em uma amostra de jovens entre 16 e 25 anos, falantes da língua inglesa e com baixo nível de leitura. $\mathrm{O}$ estudo usou duas medidas de vocabulário, uma de amplitude (o teste PPVT) e outra de profundidade (o subteste de vocabulário do WISC-III). Ambas as medidas estavam fortemente correlacionadas entre si $(r=80)$. $\mathrm{Na}$ análise correlacional com as habilidades de leitura, os valores dos coeficientes das medidas do vocabulário foram mais altos com as medidas de compreensão linguística e compreensão textual do que com a habilidade de reconhecer palavras. $\mathrm{Na}$ análise de regressão e das variáveis latentes, evidenciou-se que as medidas de vocabulário podem ser consideradas com um componente da compreensão linguística, e não um contribuinte independente da compreensão de texto. Esses dados corroboram com a ideia de que não há necessidade de um componente adicional, o vocabulário, no modelo simples de leitura.

Já na outra teoria de leitura, a Hipótese de Qualidade Lexical (Perfetti \& Stafura, 2014), o léxico é considerado o componente diferenciador do nível de compreensão textual. O processamento lexical inclui a forma (sistema ortográfico), os sons (informações fonológicas) e os significados (semântica) das palavras. A qualidade e a rapidez do resgate das palavras na leitura permitirão ao leitor fazer as integrações necessárias dos significados das palavras lidas, para construir o significado do texto.

Isso quer dizer que a compreensão textual é suportada pelos conhecimentos que permitem ao leitor ler e entender as palavras. Isso inclui a precisão e a extensão (quantidade) com que as representações ortográficas, fonológicas e semânticas estão armazenadas na memória de curto e longo prazo. O vocabulário, nesse caso, seria uma medida semântica que ajudaria o leitor a ler as palavras e, consequentemente, o texto (Perfetti \& Stafura, 2014). Por exemplo, o erro no processamento das homônimas homófonas acender (pôr fogo) e ascender (subir) podem comprometer as precisões de significados, pelo fato de serem palavras parecidas ortográfica e fonologicamente.

A maioria dos estudos sobre leitura advém de falantes da língua inglesa. O inglês é uma língua com muita complexidade ortográfica nas relações entre os sons da fala e os grafemas. As crianças falantes de línguas cuja ortografia é mais regular na relação entre letra e som parecem adquirir a leitura de forma mais fácil e com menos tempo do que aquelas que se utilizam de ortografias com maior consistência nas correspondências de grafemas e fonemas (Seymour, Aro, \& Erskine, 2003).

Tendo em vista a variação da ortografia em diferentes línguas, estudo em ortografia mais regular, como o grego (Protopapas, Mouzaki, Sideridis, Kotsolakou, \& Simos, 2013), mostrou evidências similares com os estudos em língua inglesa (Braze et al., 2007; Braze et al., 2016; Ouellette \& Beers, 2010; Tunmer \& Chapman, 2012). Protopapas, Mouzaki, Sideridis, Kotsolakou e Simos, (2013) encontraram uma relação de predição das medidas de vocabulário com a compreensão de texto, como em Braze et al. (2007), Braze et al. (2016), Ouellette e Beers (2010) e Tunmer e Chapman (2012). No estudo de Protopapas et al. (2013), o vocabulário, composto pelas medidas do WISC-III e PPVT, explicou $7,8 \%$ da compreensão de texto, depois de controlar a influência de algumas variáveis (idade, inteligência não verbal, compreensão linguística, fluência e leitura de palavras e de pseudopalavras). $\mathrm{Na}$ análise longitudinal, um ano depois, o vocabulário explicou $4,6 \%$ da compreensão de texto.

Ainda Protopapas et al. (2013) discutem o papel do vocabulário como medida de compreensão linguística, haja vista que, na análise de equação estrutural, as medidas de vocabulário tiveram uma relação direta com a compreensão linguística. O interessante é que Protopapas et al. (2013) com em Braze et al. (2016) utilizaram, além de uma medida de amplitude de vocabulário (PPVT - Peabody Picture Vocabulary Test), um teste de profundidade de vocabulário (vocabulário do WISC-III).

O português é uma língua com maior consistência nas correspondências grafema-fonema em relação ao inglês. Um estudo nacional de Piccolo e Salles (2013) explorou a relação entre desempenho de 
leitura, memória de trabalho e vocabulário em uma amostra de crianças do $3^{\circ}$ ao $6^{\circ}$ ano escolar. O teste de vocabulário do WISC foi a medida de vocabulário. $\mathrm{Na}$ análise estatística de regressão, os resultados mostraram que o vocabulário sozinho explicou 14\% da variância da leitura de palavras isoladas, 14\% da leitura de palavras irregulares, $28 \%$ da compreensão de texto (reconto oral) e $28 \%$ da compreensão de texto (resposta oral a questões do texto). Esses achados informam que, embora haja uma relação entre o vocabulário do WISC e as medidas de leitura, ainda há muito a se verificar dessa relação no contexto de crianças falantes do português.

O presente estudo pretende verificar de que maneira o vocabulário está relacionado com a compreensão de texto e a leitura de palavras, mesmo depois de controlar a influência de algumas habilidades linguísticas (consciência fonológica e morfológica) e cognitiva (memória de curto-prazo). Estudo mostram que a consciência fonológica e a memória de curto prazo fazem parte do processamento fonológico, estratégia chave para o sucesso na leitura de palavras (Justi \& Roazzi, 2012). A consciência morfológica também tem sido evidenciada como habilidade linguística requerida na leitura de palavras (Guimarães \& Mota, 2016), na compreensão de texto (Deacon, Tong, \& Francis, 2015) e relacionada ao vocabulário (Sparks \& Deacon, 2015).

Nesse sentido, este estudo explora duas questões. A primeira é se há uma relação do subteste de vocabulário do WISC- III com a precisão de leitura de palavras, como é apontado pela teoria HQL. Essa é uma questão importante, pois a HQL postula que o conhecimento sobre o significado da palavra, componente semântico, é um dos recursos de recuperação da palavra na leitura. Nesse sentido, espera-se que, mesmo retirando a influência do processamento fonológico (consciência fonológica e memória de curto prazo) e da consciência morfológica, esse tipo de vocabulário (profundo) ainda ajudaria que as crianças lessem as palavras de maneira precisa. A segunda questão é se subteste de vocabulário do WISC- III contribuiria para a compreensão de texto, depois do controle da influência da precisão na leitura de palavras, na consciência fonológica, na memória de curto prazo e na consciência morfológica. Nesse sentido, pode-se hipotetizar a contribuição do vocabulário para a compreensão de texto via compreensão linguística, como mostram os estudos (Braze et al., 2016; Protopapas et al., 2013; Tunmer \& Chapman, 2012).

\section{Método}

\section{Participantes}

Os participantes pertenciam ao projeto "Avaliação Psicométrica de medidas de consciência metalinguística", do Departamento de Psicologia da Universidade Federal de Juiz de Fora (UFJF), submetido ao Comitê de Ética e Pesquisa da UFJF - protocolo de número 1683.027.2009. Todas as crianças participaram com o consentimento dos responsáveis, por meio da assinatura do Termo de Consentimento Livre e Esclarecido. As crianças também foram elucidadas sobre a participação voluntária na pesquisa. Pelo critério de conveniência, foram recrutadas 174 crianças, entre 8 a 11 anos de idade (104 meninas), em três escolas públicas de Minas Gerais. Dessas, 85 estavam matriculadas no $3^{\circ}$ ano e 89 no $4^{\circ}$ ano do Ensino Fundamental.

\section{Instrumentos}

\section{Consciência Fonológica}

Roteiro de avaliação da consciência fonológicaRACF (Santos, 1996). A tarefa avalia a identificação dos fonemas ou "som inicial", "final" e "do meio" das palavras. Cada item é composto de uma palavra modelo e outras três, para alternativas de resposta. Foi atribuído um ponto para cada acerto e zero para erro, sendo a pontuação máxima possível 15.

A tarefa tem evidência de validade preditiva demonstrada no estudo de Santos (1996), validade de critério, por ano escolar (Suehiro \& Santos, 2011) e nível de leitura (Mota, Santos, Guimarães, \& Conti, 2014). Outras propriedades psicométricas, como a validade de construto, também foram testadas com o RACF (Santos \& Lima, 2017). Segundo Santos (1996), a análise de consistência interna da tarefa apresenta índices adequados, alfa de Cronbach $(\alpha)>0,70 . \mathrm{Na}$ amostra deste estudo $(n=174)$, o valor da consistência interna pode ser considerado moderado, com carência, $\alpha=0,65$.

\section{Consciência Morfológica}

Analogia de Palavras (Nunes, Bindman, \& Bryant, 1997, adaptado por Mota, 1996). A tarefa analisa a capacidade da criança em manejar os morfemas. Em cada item da tarefa, a criança deverá ser capaz de formar uma palavra morfologicamente complexa a partir de uma palavra-alvo. Por exemplo, tênis-tenista; arte-? A tarefa é composta de oito itens com morfemas derivacionais. Foi atribuído um ponto para cada acerto e zero para erro. 
Segundo Mota, Santos e Guimarães (2014), a tarefa apresenta validade de critérios por ano escolar e como índices moderados com carência para a morfológica derivacional $(\alpha=0,59)$. $\mathrm{Na}$ amostra deste estudo $(n=174)$, a análise de consistência da tarefa foi baixa, $\alpha=0,47$.

\section{Vocabulário}

Subteste de Vocabulário - WISC III (Wechsler, 2002). Esse subteste é uma medida do vocabulário no qual a criança é questionada a definir as palavras oralmente. São 30 palavras apresentadas, uma a uma, para a criança explicá-las. Os escores ponderados foram selecionados para a realização das análises estatísticas. Os coeficientes de fidedignidade para esse subteste foram calculados pelo do método de Guttmam e mostram bons índices de consistência interna igual a 0,79 , de acordo como manual (Wechsler, 2002).

\section{Memória de Curto Prazo}

Subteste de Dígito - ordem versa e inversa - WISC III (Wechsler, 2002). O subteste foi aplicado segundo as instruções expressas em seus manuais. A pontuação total da ordem versa e inversa foi somada e transformada em escores ponderados. Os escores ponderados foram selecionados para a realização das análises estatísticas. O coeficiente de fidedignidade para esse subteste foi calculado pelo método de Guttmam, apresentando bons índices de consistência interna (Wechsler, 2002).

\section{Precisão de Leitura de Palavras}

Subteste de leitura do Teste de Desempenho Escolar - TDE (Stein, 1994). O subteste verifica a precisão da leitura em voz alta de um conjunto de 70 palavras isoladas do contexto distribuídas em uma única folha. O subteste foi administrado e corrigido de acordo com as instruções e os procedimentos especificados no manual. Um ponto foi dado para cada acerto na leitura da palavra e zero ponto para cada erro. A tarefa apresenta evidência de validade de critério e bom índice de consistência interna, $\alpha>0,70$ (Stein, 1994).

\section{Compreensão de Texto}

Teste de Cloze (Santos 2005). Consiste em um texto do qual se suprimem alguns vocábulos, pedindo ao leitor que preencha os espaços com as palavras que melhor completarem o sentido do texto. Nesta pesquisa, foram utilizados dois textos: "Uma vingança infeliz" e "A princesa e o fantasma", elaborados por Santos
(2005) para serem utilizados com crianças da faixa etária do Ensino Fundamental. Ambos os textos são semelhantes na extensão (204 palavras), mas com níveis de dificuldades diferentes (Santos,2005). Para correção, foi escolhida a literal, que considera correta somente a resposta que contém a palavra idêntica omitida. Sendo assim, foram rejeitadas respostas com sinônimos ou palavras escritas de forma incorreta ortograficamente. A adoção desse método de correção pretendeu impedir que critérios subjetivos permeassem a correção. A pontuação máxima em cada texto é de 15 pontos, número referente à quantidade de lacunas. Os dois textos junto perfazem um total de 30 pontos.

Segundo Suehiro e Santos (2015), as histórias apresentam evidências de validade de critério, por ano escolar, bem como indicadores de consistência interna dentro dos padrões considerados adequados, $\operatorname{com} \alpha>$ 0,80 . $\mathrm{Na}$ amostra do estudo $(n=174)$, o índice de correlação entre os dois textos do Cloze, "Uma vingança infeliz" e "A princesa e o fantasma", foi significativo, moderado e positivo $(r=0,53 ; p=<0,001)$. A correlação de cada texto com uma medida patronizada de leitura (TDE) foi significativa, positiva e moderada com o texto "A princesa e o fantasma" $(r=0,55, p<0,01) \mathrm{e}$ "Uma vingança infeliz" $(r=0,48, p<0,01)$.

\section{Procedimento}

As aplicações das tarefas e dos testes selecionados ocorreram no segundo semestre escolar do ano de 2010, na própria escola das crianças. Todas aconteceram em duas sessões, uma individual e outra coletiva. $\mathrm{Na}$ primeira sessão, aplicaram-se os subtestes do vocabulário - WISCIII, dígito - WISCIII, de leitura - TDE, sob orientação do manual de aplicação e das tarefas de consciência fonológica e morfológica. Para aplicação do roteiro de avaliação da consciência fonológica (RACF), falou-se para a criança uma palavra modelo e depois outras três, para que ela indicasse quais dessas três começavam com o mesmo som da palavra modelo. Na tarefa de analogia derivacional, foi apresentado à criança um par de palavras relacionadas entre si, depois ela ouviu mais uma palavra e deveria completar com outra. $\mathrm{Na}$ segunda sessão, ocorreu a aplicação coletiva dos testes de Cloze. Foram entregues para cada criança os textos "Uma vingança infeliz" e "A princesa e o fantasma" com a seguinte instrução: "Eu vou dar para você duas estórias em que estão faltando alguns pedacinhos. Eu gostaria que você lesse as estórias e depois completasse os pedacinhos que estão faltando, mas lembre-se de que você só pode completar com apenas uma palavinha". 


\section{Análise de Dados}

Os dados foram organizados no pacote estatístico Statistical Package for Social Science for Windows (SPSS) (versão 13.0). Primeiramente, foram efetuadas análises de correlação $r$ de Pearson entre os indicadores de habilidades de leitura, vocabulário, linguísticas (consciência fonológica e morfológica) e cognitiva (memória de curto prazo). Para verificar o valor preditivo do vocabulário com as habilidades de leitura, foram realizadas duas análises de regressão hierárquica por ano escolar $\left(3^{\circ}\right.$ e $4^{\circ}$ anos), para examinar o quanto o vocabulário pode contribuir para explicar a variância dos escores de leitura de palavras e de compreensão de texto.

\section{Resultados}

A Tabela 1 exibe as estatísticas descritivas das medidas utilizadas no estudo. Antes de conduzir mais análises, foram examinadas as propriedades distributivas das medidas pela divisão da skewness/DP (Field, 2009). A pontuação do teste de precisão de leitura de palavras (TDE-Leitura) teve uma distribuição negativa e assimétrica. A transformação logarítmica foi utilizada para alcançar a normalidade (Field, 2009). A pontuação transformada foi usada nas análises posteriores.

As correlações entre as medidas do estudo por ano escolar $\left(3^{\circ}\right.$ e $4^{\circ}$ anos) são relatadas na tabela 2. A magnitude dos coeficientes de correlação do vocabulário foi moderada, positiva e significativa, com a compreensão de texto $\left(3^{\circ}\right.$ ano $r=0,48, p<0,01 ; 4^{\circ}$ ano $r=0,48, p<0,01)$, e fraca, positiva e significativa, com a precisão de leitura de palavras $\left(3^{\circ}\right.$ ano $r=0,31, p<$ 0,$01 ; 4^{\circ}$ ano $\left.r=0,24, p<0,01\right)$. As outras medidas cognitivas também correlacionaram significativamente, de maneira fraca e moderada, com as medidas de leitura. A verificação da matriz de correlação sugere que há correlações mais fortes entre o vocabulário com a compreensão de texto do que com a precisão de leitura de palavras.

As análises de regressão hierárquicas foram conduzidas para verificar, (a) se o vocabulário prediz o reconhecimento de palavras, mesmo depois da consciência fonológica, morfológica e a memória de curto prazo forem controladas; (b) se o vocabulário prediz a compreensão de texto, mesmo após o controle da influência da consciência fonológica, morfológica, memória de curto prazo e precisão de leitura de

Tabela 1.

Estatística Descritiva das Medidas por Ano Escolar

\begin{tabular}{|c|c|c|c|c|c|c|c|c|}
\hline \multirow{2}{*}{$\begin{array}{l}\text { Medidas } \\
\text { (Máximo de } \\
\text { pontuação) }\end{array}$} & \multicolumn{4}{|c|}{$\begin{array}{c}3^{\circ} \text { ano } \\
(n=85)\end{array}$} & \multicolumn{3}{|c|}{$\begin{array}{c}4^{\circ} \text { ano } \\
(n=89)\end{array}$} & \multirow[b]{2}{*}{ Skewness/DI } \\
\hline & $M$ & $D P$ & $95 \%$ IC & Skewness/DP & M & $D P$ & $95 \%$ IC & \\
\hline Idade em meses & 102 & 9,7 & $99,9-104,7$ & & 111,5 & 6 & $110-112,8$ & \\
\hline CF (15) & 11,3 & 2,6 & $10,8-11,9$ & $\begin{array}{c}0,44 / \\
0,26\end{array}$ & 12,3 & 2,1 & $11,8-12,7$ & $\begin{array}{c}0,41 / \\
0,25\end{array}$ \\
\hline $\mathrm{CM}(8)$ & 4,7 & 1,4 & $4,5-5,1$ & $\begin{array}{c}0,05 / \\
0,26\end{array}$ & 5,2 & 1,3 & $4,9-5,5$ & $\begin{array}{c}0,11 / \\
0,25\end{array}$ \\
\hline $\mathrm{MCP}^{\mathrm{a}}(19)$ & 11,2 & 3,1 & $10,5-11,9$ & $\begin{array}{c}0,27 / \\
0,26\end{array}$ & 12 & 3,8 & $11,2-12,8$ & $\begin{array}{c}0,44 / \\
0,25\end{array}$ \\
\hline $\operatorname{VOC}^{\mathrm{a}}(19)$ & 10,9 & 3,4 & $10,2-11,6$ & $\begin{array}{c}0,26 / \\
0,26\end{array}$ & 10,9 & 3,4 & $10,2-11,6$ & $\begin{array}{c}0,31 / \\
0,25\end{array}$ \\
\hline PLP (70) & 61,1 & 8,8 & $59,2-63$ & $\begin{array}{l}2,7 / \\
0,26\end{array}$ & 63,6 & 4,9 & $62,5-64,6$ & $\begin{array}{l}-1,4 / \\
0,25\end{array}$ \\
\hline СТ (30) & 8,4 & 5,1 & $7,3-9,6$ & $\begin{array}{c}0,31 / \\
0,26\end{array}$ & 10,7 & 5,1 & $9,7-11,8$ & $\begin{array}{c}-0,96 / \\
0,25\end{array}$ \\
\hline
\end{tabular}

Nota. CF- consciência fonológica (Roteiro de Consciência Fonológica); CM - consciência morfológica (Analogia de Palavras); MCP - memória de curto prazo (Dígito/WISC-III); VOC - vocabulário (Vocabulário/WISC-III); PLP - precisão de leitura de palavra (TDE/leitura); CT - compreensão de texto (Cloze).

${ }^{\text {a }}$ Dados ponderados. 
Tabela 2.

Correlação das Medidas Usadas no Estudo

\begin{tabular}{|c|c|c|c|c|c|c|c|}
\hline Ano & Medidas & Idade & $\mathrm{CF}$ & $\mathrm{CM}$ & MCP & VOC & PLP \\
\hline \multirow[t]{7}{*}{$3^{\circ}$ ano } & 1- Idade & & & & & & \\
\hline & 2-CF & $0,26^{*}$ & & & & & \\
\hline & $3-\mathrm{CM}$ & 0,48 & $0,34 * *$ & & & & \\
\hline & $4-\mathrm{MCP}^{\mathrm{a}}$ & $0,18^{*}$ & $0,34 * *$ & $0,19 *$ & & & \\
\hline & $5-\mathrm{VOC}^{\mathrm{a}}$ & 0,48 & $0,30 * *$ & $0,23^{*}$ & $0,24 *$ & & \\
\hline & 6-PLP & $0,41 * *$ & $0,48 * *$ & $0,25^{*}$ & $0,34^{*}$ & $0,31 * *$ & \\
\hline & 7-CT & $0,26 * *$ & $0,49 * *$ & $0,34 * *$ & $0,46^{*}$ & $0,48 * *$ & $0,59 * *$ \\
\hline \multirow[t]{7}{*}{$4^{\circ}$ ano } & 1-Idade & & & & & & \\
\hline & 2-CF & $0,26^{*}$ & & & & & \\
\hline & 3-CM & 0,05 & $0,34 * *$ & & & & \\
\hline & $4-\mathrm{MCP}^{\mathrm{a}}$ & $0,25^{*}$ & $0,35^{* *}$ & 0,19 & & & \\
\hline & $5-\mathrm{VOC}^{\mathrm{a}}$ & 0,18 & $0,31 * *$ & $0,23^{*}$ & $0,24^{*}$ & & \\
\hline & 6-PLP & $0,41 * *$ & $0,48^{* *}$ & $0,38 * *$ & $0,38 * *$ & $0,31 * *$ & \\
\hline & 7-CT & $0,26^{* *}$ & $0,49 * *$ & $0,46 * *$ & $0,46^{* *}$ & $0,48^{* *}$ & $0,59 * *$ \\
\hline
\end{tabular}

Nota. CF- consciência fonológica (Roteiro de Consciência Fonológica); CM - consciência morfológica (Analogia de Palavras); MCP - memória de curto prazo (Dígito/WISC-III); VOC - vocabulário (Vocabulário/WISC-III); PLP - precisão de leitura de palavra (TDE/leitura); CT - compreensão de texto (Cloze).

${ }^{a}$ Dados ponderados

$*<0,05 ; * *<0,01 ; * * *<0,001$

palavras. $\mathrm{Na}$ análise de regressão, a idade também esteve relacionada com as variáveis de leitura, por isso, ela será colocada como uma das variáveis de controle na análise de regressão.

Para averiguar se o vocabulário prediz o reconhecimento de palavras, mesmo depois da consciência fonológica, morfológica e memória de curto prazo, foram realizadas duas análises de regressão hierárquica para cada ano escolar. Em cada uma das regressões hierárquicas, foram realizados os seguintes passos: $1^{\circ}$ passo: a idade; $2^{\circ}$ passo: a consciência fonológica (Roteiro de consciência fonológica), a consciência morfológica (Analogia de palavras), a memória de curto prazo (Dígito/WISCIII) e $3^{\circ}$ passo: o vocabulário/ WISCIII. Os resultados das análises de regressão são apresentados na tabela 3 . O vocabulário não fez uma contribuição significativa para crianças do $3^{\circ}$ ano $(p=$ $0,20)$, tampouco no $4^{\circ}$ ano $(p=0,52)$. Esse resultado mostra o que explicou o vocabulário não explicou a leitura de palavras.

Para verificar se o vocabulário prediz a compreensão de texto, mesmo depois do controle de habilidades linguísticas e cognitivas, foram realizadas duas análises de regressão hierárquica para cada ano escolar. Em cada uma das regressões hierárquicas, foram realizados as seguintes etapas: $1^{\circ}$ passo: a idade; $2^{\circ}$ passo: a consciência fonológica (Roteiro de consciência fonológica), consciência morfológica (Analogia de palavras), memória de curto prazo (Dígito/WISC-III) e TDE/leitura e $3^{\circ}$ passo: o vocabulário/WISC-III. Os resultados das análises de regressão são apresentados na tabela 4 .

As análises de regressão mostraram que 6\% ( $3^{\circ}$ ano $)$ e $9 \%$ ( $4^{\circ}$ ano $)$ da proporção da variância vocabulário explicam a compreensão de texto, medida pelo Cloze. Isso quer dizer que o vocabulário teve uma contribuição específica com a compreensão de texto.

\section{Discussão}

O presente estudo analisou a relação do subteste de vocabulário WISC-III com as habilidades de leitura no nível da palavra e do texto, em crianças brasileiras do $3^{\circ}$ e $4^{\circ}$ anos do Ensino Fundamental. Consistente 
Tabela 3.

Resumo dos Resultados das Análises de Regressão Hierárquica do Vocabulário e Precisão de Leitura de Palavras por Ano Escolar

\begin{tabular}{|c|c|c|}
\hline \multirow{2}{*}{ Passos / Preditor } & \multicolumn{2}{|c|}{ Precisão de leitura de palavras } \\
\hline & $\beta$ & $\Delta \mathrm{R}^{2}$ \\
\hline \multicolumn{3}{|l|}{ Medidas do $3^{\circ}$ ano } \\
\hline $1^{\circ}$ Idade & $0,41 * * *$ & $0,17 * * *$ \\
\hline $2^{\circ}$ Consciência fonológica (RACF) & $0,40^{* * *}$ & $0,17 * * *$ \\
\hline Consciência morfológica (AP) & $0,23 *$ & \\
\hline Memória de curto prazo (dígito/WISC-III) & $0,25^{*}$ & \\
\hline $3^{\circ}$ Vocabulário $^{a}$ (WISC-III) & 0,12 & 0,13 \\
\hline \multicolumn{3}{|l|}{ Medidas do $4^{\circ}$ ano } \\
\hline $1^{\circ}$ Idade & $0,23^{*}$ & $0,05^{*}$ \\
\hline $2^{\circ}$ Consciência fonológica (RACF) & $0,43^{* * *}$ & $0,21 * * *$ \\
\hline Consciência morfológica (AP) & $0,22 *$ & \\
\hline Memória de curto prazo (dígito/WISC-III) & $0,42^{* *}$ & \\
\hline $3^{\circ}$ Vocabulário $^{\mathrm{a}}$ (WISC-III) & 0,07 & 0,004 \\
\hline
\end{tabular}

Nota. RCF- Roteiro de Consciência Fonológica; AP - Analogia de Palavras.

${ }^{a}$ Dados ponderados.

$*<0,05 ; * *<0,01 ; * * *<0,001$

Tabela 4.

Resumo dos Resultados das Análises de Regressão Hierárquica do Vocabulário e Compreensão de Texto por Ano Escolar

\begin{tabular}{|c|c|c|}
\hline \multirow{2}{*}{ Passos/Preditor } & \multicolumn{2}{|c|}{ Compreensão de texto } \\
\hline & $\beta$ & $\Delta \mathrm{R}^{2}$ \\
\hline \multicolumn{3}{|l|}{ Medidas do $3^{\circ}$ ano } \\
\hline $1^{\circ}$ Idade & $0,26^{*}$ & $0,07 *$ \\
\hline $2^{\circ}$ Consciência fonológica (RACF) & $0,45^{* * *}$ & $0,30 * * *$ \\
\hline Consciência morfológica (AP) & $0,33^{* *}$ & \\
\hline Memória de curto prazo (Dígito/WISC-III) & $0,42^{* * *}$ & \\
\hline $3^{\circ}$ Precisão de leitura de palavras (TDE/Leitura) & $0,39 * * *$ & $0,10 * * *$ \\
\hline $4^{\circ}$ Vocabulário $^{\mathrm{a}}$ (WISC-III) & $0,26 * *$ & $0,06^{* *}$ \\
\hline \multicolumn{3}{|l|}{ Medidas do $4^{\circ}$ ano } \\
\hline $1^{\circ}$ Idade & 0,19 & 0,04 \\
\hline $2^{\circ}$ Consciência fonológica (RACF) & $0,59 * * *$ & $0,37 * * *$ \\
\hline Consciência morfológica (AP) & 0,30 & \\
\hline Memória de curto prazo (Dígito/WISC-III) & $0,49 * * *$ & \\
\hline $3^{\circ}$ Precisão de leitura de palavras (TDE/Leitura) & $0,38^{* * *}$ & $0,11 * * *$ \\
\hline $4^{\circ}$ Vocabulário $^{\mathrm{a}}$ (WISC-III) & $0,34 * *$ & $0,09 * * *$ \\
\hline
\end{tabular}

Nota. RCF- Roteiro de Consciência Fonológica; AP - Analogia de Palavras.

${ }^{a}$ Dados ponderados.

$*<0,05 ; * *<0,01 ; * * *<0,001$ 
com as pesquisas (Protopapas et al., 2013; Tunmer \& Chapman, 2012), a análise correlacional mostrou uma associação do vocabulário com as habilidades de leitura. Ele exerceu influência maior na compreensão de texto do que na habilidade de reconhecimento de palavras. Quando se buscou quantificar as proporções das variâncias das habilidades de leitura atribuídas ao vocabulário, essa relação ficou mais evidente.

A primeira hipótese do estudo foi se o vocabulário estaria relacionado à precisão de leitura de palavras. O resultado mostrou que o vocabulário do WISC não explicou a variância da leitura de palavras das crianças. Isso pode ter uma explicação nas diferentes dimensões que o vocabulário pode abranger na leitura. $\mathrm{O}$ subteste de vocabulário do WISC-III afere um conhecimento semântico, no qual não basta só reconhecer a palavra, mas ter a competência de descrevê-la. Esse tipo de profundidade semântica pode ser menos requerida na leitura da palavra. Para ler corretamente uma palavra, a criança não necessariamente precisa ter um conhecimento mais abrangente do significado da palavra. Por exemplo, ela pode ler a palavra bicicleta (um dos itens do teste de vocabulário do WISC), mas não saber explicar o conceito dessa palavra.

Outra questão foi com as variáveis de controle utilizadas na presente pesquisa. Em outro estudo nacional de Piccolo e Salles (2013), foi encontrada uma relação entre leitura de palavras o teste de vocabulário do WISC. No entanto, as autoras não controlaram habilidades metalinguísticas com a consciência fonológica e morfológica, como neste estudo, em que as variáveis de controle juntas explicaram $17 \%$ ( $3^{\circ}$ ano) e $21 \%$ ( $4^{\circ}$ ano) da variância da leitura de palavras. Isso pode indicar que, quando retirada a influência dos componentes dos aspectos fonológicos (consciência fonológica e memória de curto prazo) e da consciência morfológica, não teve força para uma contribuição independente do vocabulário. A influência da consciência morfológica é importante, pois tal habilidade tem valor preditivo no estudo em português para a leitura de palavras e não para a compreensão de texto (Guimarães \& Mota, 2016).

A segunda hipótese do estudo foi se o subteste de Vocabulário/WISC-III contribuiria para a compreensão de texto, mesmo controlando a precisão da leitura de palavras e das variáveis de controle. O resultado mostrou que o subteste de Vocabulário/WISC-III teve uma relação com a compreensão de texto e que ela não perpassa pela precisão da leitura de palavras. Sua contribuição foi independente da influência das variáveis controladas (consciência fonológica, memória de trabalho, consciência morfológica e leitura de palavras). Isso indica que as crianças que foram melhores na pontuação no subteste de vocabulário do WISC-III também o foram na compreensão de texto.

Os achados deste estudo sugerem que o vocabulário que afere um nível de conhecimento semântico mais profundo está subjacente à compreensão de texto e não na leitura de palavras. A compreensão de texto depende do significado das palavras e a integração desse significado com um modelo mental do texto (Perfetti \& Stafura, 2014). A relação entre profundidade de vocabulário e a compreensão de texto também foi evidenciada em outros estudos (Ouellette, 2006; Braze et al., 2007; Protopapas et al. 2013).

Este estudo, evidenciou uma relação específica entre vocabulário com a compreensão de texto. Essa relação foi independente da leitura de palavras. Isso levanta a hipótese, dentro do modelo simples de leitura, de que o subteste do vocabulário do WISC-III possa ser um componente para medir a compreensão linguística, como propõem Braze et al. (2016), Protopapas et al. (2013) e Tunmer e Chapman (2012). Entretanto, pelo fato deste estudo não apresentar uma medida de compreensão linguística, parece prudente não interpretar essa ideia como uma afirmação, e sim como uma suposição para estudos futuros.

Com relação à teoria da Hipótese da Qualidade lexical, o resultado da contribuição do vocabulário para a compreensão textual, sugerem que o léxico esteja envolvido, como demonstram a proporção da variância das medidas de controle. O grupo das habilidades de consciência fonológica, morfológica e a memória de curto prazo explicaram $30 \%$ ( $3^{\circ}$ ano) $37 \%$ ( $4^{\circ}$ ano) e a precisão na leitura de palavras $10 \%$ ( $3^{\circ}$ ano) $9 \%$ (4 ano) a compreensão de leitura. Essas habilidades de alguma forma ajudam as crianças no vocabulário.

Apesar do estudo ir ao encontro das evidências científicas que apontam a relação do vocabulário com a compreensão de texto (Braze et al., 2007; Braze et al., 2016; Perfetti \& Stafura, 2014; Protopapas et al., 2013; Ricketts et al., 2007; Tunmer \& Chapman, 2012; Verhoeven \& van Leeuwe, 2008), ainda há muito a ser investigado nessa relação. O papel da semântica precisa ser melhor explorada. Primeiramente em relação às diferentes dimensões do vocabulário. Anderson e Freebody (1981) fizeram uma distinção entre duas dimensões de conhecimento de vocabulário: amplitude e profundidade. Neste estudo, utilizou-se apenas uma medida de profundidade, mas diferentes medidas 
são necessárias para que se compreenda em quais níveis e direções vocabulário se relaciona no nível da palavra e texto.

O segundo ponto seria em relação aos compreendedores de texto menos habilidosos. Muitos estudos mostraram que fracos compreendedores têm dificuldades específicas no aspecto semântico (Nation \& Snowling, 1998; Ricketts et al., 2007). Nesse sentido, diferentes desenhos metodológicos buscando validar a contribuição do vocabulário nas habilidades de leitura precisam ainda ser realizados nas pesquisas com falantes do português. Por exemplo, um recorte maior dos anos inicias da alfabetização dentro de desenho longitudinal poderia verificar o desenvolvimento do vocabulário em relação a compreensão.

\section{Conclusão}

Os achados deste estudo sugerem que a habilidade verbal medida no subteste de vocabulário do WISC-III estão atreladas à capacidade de compreensão de texto medido pelo teste de Cloze. Essa descoberta é importante tanto na campo de pesquisa como educacional, entretanto alguns aspectos limitaram o estudo. Um deles está na utilização de uma única medida de compreensão de texto e de vocabulário. Ambas as habilidades são completas e com especificações que precisam ser levadas em conta (ver Anderson e Freebody, 1981; Kendeou, Papadopoulos, \& Spanoudis, 2012). No caso do vocabulário, medidas de amplitude e profundida poderia ser utilizadas, para verificar há uma variação de contribuição dessas habilidades na leitura. Na compreensão de texto, estudo indica que distintos tipos de testes requerem diferentes habilidades subjacentes. Essas limitações são importante, sugerindo de serem consideradas em estudos futuros.

A despeito dessas limitações, pode-se considerar que o estudo trouxe implicações teóricas e práticas nas quais reforçam a importância do processamento de nível mais profundo do significado das palavras para compreender um texto. O papel da profundidade do vocabulário na compreensão de leitura sugere que a compreensão pode se beneficiar do ensino focado na profundidade do conhecimento das palavras e na organização semântica. Estudos de intervenção que incorporasse exercícios para aumentar o tamanho do vocabulário, bem como a profundidade da semântica e sua organização poderiam esclarecer as possibilidades da influência das dimensões do vocabulário na leitura

\section{Referências}

Anderson, R. C., \& Freebody, P. (1981). Vocabulary knowledge. Em J. Guthrie (Ed.), Comprehension and Teaching: Research Reviews (pp. 77-117). Newark, DE: International Reading Association.

Beck, I. L., Perfetti, C., \& McKeown, M. G. (1982). Effects of long-term vocabulary instruction on lexical access and reading comprehension. Journal of Educational Psychology, 74(4), 506-521. doi:10.1037/0022-0663.74.4.506

Braze, D., Tabor, W., Shankweiler, D. P., \& Mencl, W. (2007). Speaking up for vocabulary: Reading skill differences in young adults. Journal of Learning Disabilities, 40(3), 226- 243. doi: 10.1177/00222194070400030401

Braze, D., Katz, L., Magnuson, J. S., Mencl, W. E., Tabor, W., Dyke, J. A. V., Gong, T., Johns, C., \& Shankweiler, D. P. (2016). Vocabulary does not complicate the Simple View of Reading. Reading and Writing, 29(3), 435- 451. doi: 10.1007/ s11145-015-9608-6

Deacon, S. H., Tong, X., \& Francis, K. (2015).The relationship of morphological analysis and morphological decoding to reading comprehension. Journal of Research in Reading, 40, 1-16. doi: 10.1111/1467-9817.12056

Elleman, A. M., Lindo, E. J., Morphy, P., \& Compton, D. L. (2009). The impact of vocabulary instruction on passage level comprehension of school-age children: A meta-analysis. Journal of Research on Educational Effectiveness, 2(1), 1-44. doi: 10.1080/19345740802539200

Field, A. (2009). Descobrindo estatística usando o SPSS ( $2^{\mathrm{a}}$ ed.). Porto Alegre: Armed.

Foorman, B. R., Herrera, S., Petscher, Y., Mitchell, A., \& Truckenmiller, A. (2015). The structure of oral language and reading and their relation to comprehension in Kindergarten through Grade 2. Reading and Writing, 28(5), 655-681. doi: 10.1007/ s11145-015-9608-6

Gough, P. B., \& Tunmer, W. E. (1986). Decoding, reading, and reading disability. RASE: Remedial \& Special Education, 7(1), 6-10. Recuperado de http://citeseerx.ist.psu.edu/viewdoc/download?doi $=10 \cdot 1 \cdot 1 \cdot 905.7606 \&$ rep $=$ rep1\&type $=$ pdf 
Guimarães, S. B., \& Mota, M. (2016). Qual a contribuição da consciência morfológica das crianças na precisão de leitura de palavras e compreensão de texto no português? Estudos de Psicologia, 21(3), 239348. doi: 10.5935/1678-4669.20160023

Holahan, J., Ferrer, E., Shaywitz, B., Rock, D., Kirsch, I., Yamamoto, K., Michaels, R., Marchione, K., \& Shaywitz, S. (2016). Growth in Reading Comprehension and Verbal Ability from Grades 1 through 9. Journal of Psychoeducational Assessment, 30(1), 1-25. doi:10.1177/0734282916680984

Hoover, W. A., \& Gough, P. B. (1990). The simple view of reading. Reading and Writing, 2(2), 127-160. doi: 10.1007/BF00401799

Justi, C., \& Roazzi, A. (2012). A contribuição de variáveis cognitivas para a leitura e a escrita no Português Brasileiro. Psicologia: Reflexão e Crítica, 25(3), 605-614. doi: 10.1590/S0102-79722012000300021

Kendeou, P., Papadopoulos, T. C., \& Spanoudis, G. (2012). Processing demands of reading comprehension tests in young readers. Learning and Instruction, 22(5), 354-367. doi: 10.1016/j. learninstruc.2012.02.001

Kim, Y. S., \& Wagner, R. K. (2015). Text (Oral) Reading fluency as a construct in reading development: An investigation of its mediating role for children from grades 1 to 4. Scientific Studies of Reading, 19, 224-242. doi: 10.1080/10888438.2015.1007375

Maluf, M. R., Zanella, M. S., \& Pagnez, K. S. M. M. (2006) . Habilidades metalinguísticas e linguagem escrita nas pesquisas brasileiras. Boletim de Psicologia, 56(124): 67-92. Recuperado de http://pepsic. bvsalud.org/scielo.php?script=sci_arttext\&pi$\mathrm{d}=$ S0006-59432006000100006\&lng=pt\&tlng=

Mota, M. (1996). Children's role of grammatical rules in spelling (Tese de doutorado não publicada). Universidade de Oxford, Inglaterra.

Mota, M., Santos, A., \& Guimarães, S. B. (2014). Evidências de validade e consistência interna de tarefas de analogia gramatical. Estudos de Psicologia, 19, 250257. doi: 10.1590/S1413-294X2014000400002

Mota, M., Santos, A, Guimarães, S. B., \& Conti, C. (2014). Evidências de validade do roteiro de avaliação da consciência fonológica. Estudos e Pesquisas em Psicologia, 14(3), 933-948. doi: 10.12957/ epp.2014.13892
Nation, K., \& Snowling, M. J. (1998). Semantic processing and the development of word recognition skills: Evidence from children with reading comprehension difficulties. Journal of Memory and Language, 39(1), 85-101. doi: 10.1006/ jmla.1998.2564

Nunes, T., Bindman, M., \& Bryant, P. (1997). Morphological strategies: Developmental stages and processes. Developmental Psychology, 33(4), 637-649. doi: $10.1037 / / 0012-1649.33 .4 .637$

Oakhill, J., Cain, K., \& Elbro, C. (2015). Understanding and teaching reading comprehension: a handbook. London: Routledge.

Ouellette, G. P. (2006). What's meaning got to do with it: The role of vocabulary in word reading and reading comprehension. Journal of Educational Psychology, 98(3), 554-566. doi: 10.1037/0022-0663.98.3.554

Ouellettte, G., \& Beers, A. (2010). A not-so-simple view of reading: How oral vocabulary and visual-word recognition complicate the story. Reading and Writing, 23(2), 189-208. doi: 10.1007/s11145-008-9159-1

Protopapas, A., Mouzaki, A., Sideridis, G., Kotsolakou, A., \& Simos, P. (2013). The role of vocabulary in the context of the simple view of reading. Reading \& Writing Quarterly, 29(2), 168-202. doi: 10.1080/10573569.2013.758569

Perfetti, C., \& Stafura, J. (2014). Word knowledge in a theory of reading comprehension. Scientific Studies of Reading, 18(1), 22-37. doi: 10.1080/10888438.2013.827687

Piccolo, L., \& Salles, J. (2013). Vocabulário e memória de trabalho predizem desempenho em leitura de crianças. Psicologia: Teoria e Prática, 15(2), 180-191. Recuperado de ttp://editorarevistas.mackenzie. br/index.php/ptp/article/viewFile/4576/4461

Ricketts, J., Nation, K., \& Bishop, D. V. M. (2007).Vocabulary is important for some, but not all reading skills. Scientific Studies of Reading, 11(3), 235-257. doi: 10.1080/10888430701344306

Santos, A. (1996). A influência da consciência fonológica na aquisição da leitura e da escrita. Em F. F. Sisto, G. C. Oliveira, L. D. T. Fini, M. T. C. C. Souza, \& R. P. Brenelli (Eds.), Atuação psicopedagógica e aprendizagem escolar (pp. 213- 247). Petrópolis: Vozes. 
Santos, A. (2005). O Teste de Cloze como instrumento de avaliação da compreensão em leitura. Relatório técnico. Itatiba: Universidade São Francisco.

Santos, A., \& Lima, T. (2017). Exploratory and confirmatory fator analysis of the Roteiro de Avaliação da Consciência Fonológica, a phonological awareness test. Estudos de Psicologia, 34(2), 211-218. doi:10.1590/1982-02752017000200003

Santos, I., Melo, M. \& Roazzi, A. (2016). Consciência fonológica e alfabetização em crianças brasileiras: Como esta relação tem evoluído? Iniciação Científica CESUMAR, 18(2), 211-221. doi 10.17765/1518-1243.2016v18n2p211-221

Seymour, P., Aro, M., \& Erskine, J. M. (2003). Foundation literacy acquisition in European orthographies. British Journal of Psychology, 94(2), 143-174. doi: 10.1348/000712603321661859

Stein, L. (1994). Teste de desempenho escolar. São Paulo: Casa do Psicólogo

Sparks E., \& Deacon S. H. (2015). Morphological awareness and vocabulary acquisition: A longitudinal examination of their relationship in English-speaking children. Applied Psycholinguistics, 36(2), 299-321. doi: 10.1017/S0142716413000246

Stanovich, K., Cunningham, A., \& Freeman, D. (1984). Intelligence, cognitive skills and early reading progress. Reading Research Quarterly, 19(3), 278-303. doi: $10.2307 / 747822$
Suehiro, A., \& Santos, A. (2011). Roteiro de Avaliação de Consciência Fonológica (RACF). Acta Colombiana de Psicología, 14(1), 147-154.Recuperado de http:// www.scielo.org.co/pdf/acp/v14n1/v14n1a13.pdf

Suehiro, A., \& Santos, A. (2015). Compreensão de leitura e consciência fonológica: Evidências de validade de suas medidas. Estudos de Psicologia, 32(2), 201211. doi:10.15 90/0103-166X2015000200005

Tunmer, W., \& Chapman, J. (2012). The simple view of reading redux: Vocabulary knowledge and the independent components hypothesis. Journal of Learning Disabilities, 45(5), 453-466. doi: $10.1177 / 0022219411432685$

Verhoeven, L., \& van Leeuwe, J. (2008). Prediction of the development of reading comprehension: A longitudinal study. Applied Cognitive Psychology, 22(3), 407-423. doi: 10.1002/acp.1414

Verhoeven, L., van Leeuwe, J., \& Vermeer, A. (2011). Vocabulary growth and reading development across the elementary school years. Scientific Studies of Reading, 15(1), 8-25. doi:10.1080/10888438.201 1.536125

Wechsler, D. (2002). WISC-III: Escala de Inteligência Wechsler para Crianças: Manual. São Paulo: Casa do Psicólogo.

Recebido em: 31/05/2018

Reformulado em: 19/10/2018 Aprovado em: 07/12/2018 
Sobre as autoras:

Silvia Brilhante Guimarães é psicóloga, pedagoga, doutora em Psicologia pela Universidade do Estado do Rio de Janeiro (UERJ), mestre em Psicologia pela Universidade Federal de Juiz de Fora (UFJF) e bolsista de pós-doutorado pelo Programa de Pós-graduação de Psicologia da Universidade Salgado de Oliveira.

ORCID: https://orcid.org/0000-0002-6578-8877

E-mail: silvia_brilhante@yahoo.com.br

Renata Mousinho é fonoaudióloga, doutora e mestre em Linguística pela Universidade Federal do Rio de Janeiro (UFRJ), com estágio pós-doutoral em Psicologia, UFRJ, especializada em Psicomotricidade (ISRP-Paris) e em Educação Inclusiva (UGF). É ainda professora associada da graduação em Fonoaudiologia da Faculdade de Medicina, da Universidade Federal do Rio de Janeiro e coordenadora do Projeto ELO: escrita, leitura e oralidade.

ORCID: https://orcid.org/0000-0002-7117-8952

E-mail: renatamousinho@ufrj.br

Contato com as autoras:

Rua Senador Vergueiro, 148, Flamengo

Rio de Janeiro-RJ, Brasil

CEP: 22230-001 